\title{
COMMENTARY
}

\section{The Serotype-Specific Role of Regulator of Cov Polymorphisms in the Pathogenesis of Invasive Group A Streptococcal Infections}

\author{
Stephan Brouwer and Mark J. Walker
}

\begin{abstract}
From the School of Chemistry and Molecular Biosciences and the Australian Infectious Diseases Research Centre, The University of Queensland, Brisbane, Queensland, Australia
\end{abstract}

Establishing meaningful relationships between virulence gene variation and infectious disease outcome is fundamental to advancing our understanding of bacterial pathogenicity. Bacterial pathogens frequently gain genetic variations that drive the adaptive evolution of virulence, often enhancing persistence in distinct host niches. However, such genotype-phenotype correlations remain incompletely understood. This is particularly true for the human pathogen Streptococcus pyogenes (group A Streptococcus; GAS) whose versatile adaptive capability is largely associated with high genome plasticity and with highly variable sets of accessory genes. ${ }^{1,2}$ Such variability in genome content also poses a major hurdle in vaccine development. ${ }^{2}$ Despite over a century of intense research, there is still no commercially available vaccine to prevent GAS infections on the market.

GAS causes a wide variety of human diseases with diverse clinical manifestations. These infections range from mild illnesses such as pharyngitis (strep throat) to severe, life-threatening invasive infections. It is estimated that more than 18 million individuals globally suffer from serious GAS infections, accounting for more than 500,000 deaths annually. ${ }^{3}$ This immense health burden places GAS among the top 10 mortality-causing infectious agents worldwide. Alarmingly, the incidence of invasive GAS infections has increased in many industrialized countries over the past few decades. ${ }^{4}$ Recently, other manifestations of GAS disease, including the toxin-mediated scarlet fever, have followed a similar trend..$^{5}$ Although the triggers for these epidemics remain unclear, the global resurgence and persistence of severe invasive GAS infections has been attributed to the circulation of highly virulent GAS strains. A significant driver for the high-virulence phenotype of invasive GAS strains is represented by genetic variations in virulencerelated genes. One of the most frequently detected mutations in invasive GAS isolates occurs in the genes encoding the control of virulence (CovRS, or CsrRS) two-component regulatory system, which plays a major role in controlling GAS virulence factor expression. ${ }^{6}$

In this issue of The American Journal of Pathology, Bernard and et $\mathrm{al}^{7}$ report the role of naturally-occurring polymorphisms in the regulator of Cov (RocA), an accessory protein to the CovRS two-component system, in the molecular pathogenesis of serotype M28 GAS invasive infections. Previous studies have revealed that rocA is unusually polymorphic in acapsular serotype M28 GAS strains recovered from patients with invasive infections, ${ }^{8,9}$ suggesting that the identified missense and nonsense mutations might confer a selective fitness advantage in vivo. To gain a better understanding of the contribution of these naturally-occurring rocA polymorphisms to invasive GAS disease, genome-wide transcriptome analysis was conducted on selected strains containing rocA polymorphisms. Of the five unique RocA variants screened, the missense mutations H60Y (which additionally carried deletion mutations in the variable number tandem repeat sequence), P97L and T442P substantially altered the transcriptome of serotype M28 GAS. Among these were several transcripts for known virulence factors, the expression of which is under the control of the CovRS two-component system. Similar

Accepted for publication July 30, 2019.

Disclosures: None declared.

Address correspondence to Mark J. Walker, Ph.D., School of Chemistry and Molecular Biosciences, The University of Queensland, Cooper Rd., St. Lucia, QLD, 4072, Australia. E-mail: mark.walker@uq.edu.au. 
expression patterns were observed in an isogenic $\Delta \operatorname{roc} A$ deletion mutant strain, suggesting important roles for these residues in RocA function. Of these rocA deletion-like mutations, the T442P RocA variant is of particular interest as it is located in the putative non-functional histidine kinase ATPase domain of RocA. Remarkably, multiple isolates independently developed this mutation during human invasive infections, indicating a significant contribution to increased virulence of serotype M28 GAS in vivo. This was confirmed in murine and nonhuman primate models of necrotizing myositis, where serotype M28 GAS displayed significantly increased virulence due to mutation at amino acid position 442 .

Naturally-occurring polymorphisms in rocA have been reported in other GAS M protein serotypes from both invasive and noninvasive infections, ${ }^{10-15}$ often resulting in protein truncation and altered expression of GAS virulence genes. For example, GAS isolates of serotype M18 are uniformly rocA mutants, which results in the unique, highly encapsulated phenotype of this serotype. ${ }^{16}$ On the whole, the empirical evidence points to a key role for RocA in GAS molecular pathogenesis and suggests that rocA polymorphisms are frequently selected during host infection. Yet the precise mechanistic mode of action of RocA has not been fully elucidated. Acting as an upstream regulator of CovR, RocA is hypothesized to be a pseudokinase affecting CovR phosphorylation and downstream regulatory activity in a CovS-dependent manner. ${ }^{15,17}$ Very recent experimental evidence demonstrates that RocA directly binds to and interacts with CovS in the streptococcal cell membrane to increase the kinase activity of CovS. The amino-terminal transmembrane domains of RocA were shown to be essential for intramembrane heterodimerization with CovS. ${ }^{18}$ In serotype M1 GAS, these transmembrane domains were also critical for RocA function. ${ }^{15,18}$ Results of the present study support this view, by highlighting an essential role for the transmembrane domain residues H60 and P97 in the regulatory activity of RocA in serotype M28 GAS. $^{7}$ The unexpected functional consequences of a missense mutation in the $\mathrm{C}$-terminal histidine kinase ATPase domain, however, question the assumption that this domain is non-functional in RocA. Interestingly, the functional requirement of this domain is not restricted to serotype M28 GAS. Previous studies showed that serotype M3 GAS isolates exhibit increased capsule expression due to the presence of frameshift mutations in the hypothetical histidine kinase ATPase domain, resulting in a truncated protein. ${ }^{10,11}$ The latest evidence indicates that the cytoplasmic domains play an important structural, non-enzymatic role in RocA function, facilitating homodimerization of RocA which is necessary for functional interaction with $\operatorname{CovS} .{ }^{18}$ Interestingly, the serotype-specific RocA truncation in M3 isolates caused RocA aggregation, which is hypothesized to impair RocA-CovS interaction. ${ }^{18}$ This observation raises the possibility that the M28-specific T442P polymorphism reported in the current study is similarly affecting RocA oligomerization. ${ }^{7}$ Based on findings of these recent studies, it is likely that residue T442 might be involved in important intermolecular interactions critical to RocA dimerization and for maintaining RocA function. Further research is needed to refine the structural and functional basis for the serotype-specific phenotype variations mediated by RocA.

The high frequency of mutations in regulator-encoding genes is a driving force in the phenotypic heterogeneity of pathogenic bacteria. In GAS, the stand-alone regulator of protease B (RopB, or Rgg), the multigene regulator in GAS (Mga) and the CovRS two-component regulatory system have been identified as hot-spots for mutation. ${ }^{19}$ Bernard et $\mathrm{al}^{7}$ also found a number of polymorphisms in genes of these regulators alongside rocA mutations in the collection of M28 isolates analyzed in this work. Polymorphisms in multiple regulator genes are likely to provide another additional layer of complexity to virulence gene regulation and illustrate the importance of understanding how genetic variations drive infection-niche tropism in GAS.

\section{References}

1. Bao YJ, Shapiro BJ, Lee SW, Ploplis VA, Castellino FJ: Phenotypic differentiation of Streptococcus pyogenes populations is induced by recombination-driven gene-specific sweeps. Sci Rep 2016, 6:36644

2. Davies MR, McIntyre L, Mutreja A, Lacey JA, Lees JA, Towers RJ, et al: Atlas of group A streptococcal vaccine candidates compiled using large-scale comparative genomics. Nat Genet 2019, 51:1035-1043

3. Ralph AP, Carapetis JR: Group A streptococcal diseases and their global burden. Curr Top Microbiol Immunol 2013, 368:1-27

4. Efstratiou A: Group A streptococci in the 1990s. J Antimicrob Chemother 2000, 45 Suppl:3-12

5. Walker MJ, Brouwer S: Scarlet fever makes a comeback. Lancet Infect Dis 2018, 18:128-129

6. Ikebe T, Ato M, Matsumura T, Hasegawa H, Sata T, Kobayashi K, Watanabe H: Highly frequent mutations in negative regulators of multiple virulence genes in group A streptococcal toxic shock syndrome isolates. PLoS Pathog 2010, 6:e1000832

7. Bernard PE, Kachroo P, Eraso JM, Zhu L, Madry JE, Linson SE, Saavedra MO, Cantu C, Musser JM, Olsen RJ: Polymorphisms in RocA contribute to the molecular pathogenesis of serotype M28 group A Streptococcus. Am J Pathol 2019, 189:2002-2018

8. Bernard PE, Kachroo P, Zhu L, Beres SB, Eraso JM, Kajani Z, Long SW, Musser JM, Olsen RJ: RocA has serotype-specific gene regulatory and pathogenesis activities in serotype M28 group A Streptococcus. Infect Immun 2018, 86:e0467-18

9. Kachroo P, Eraso JM, Beres SB, Olsen RJ, Zhu L, Nasser W, Bernard PE, Cantu CC, Saavedra MO, Arredondo MJ, Strope B, Do H, Kumaraswami M, Vuopio J, Grondahl-Yli-Hannuksela K, Kristinsson KG, Gottfredsson M, Pesonen M, Pensar J, Davenport ER, Clark AG, Corander J, Caugant DA, Gaini S, Magnussen MD, Kubiak SL, Nguyen HAT, Long SW, Porter AR, DeLeo FR, Musser JM: Integrated analysis of population genomics, transcriptomics and virulence provides novel insights into Streptococcus pyogenes pathogenesis. Nat Genet 2019, 51:548-559

10. Lynskey NN, Turner CE, Heng LS, Sriskandan S: A truncation in the regulator RocA underlies heightened capsule expression in serotype M3 group A streptococci. Infect Immun 2015, 83:1732-1733

11. Miller EW, Pflughoeft KJ, Sumby P: Reply to "A truncation in the regulator RocA underlies heightened capsule expression in serotype M3 group A streptococci”. Infect Immun 2015, 83:1734 
12. Miller EW, Danger JL, Ramalinga AB, Horstmann N, Shelburne SA, Sumby P: Regulatory rewiring confers serotypespecific hyper-virulence in the human pathogen group A Streptococcus. Mol Microbiol 2015, 98:473-489

13. Zhu L, Olsen RJ, Horstmann N, Shelburne SA, Fan J, Hu Y, Musser JM: Intergenic variable-number tandem-repeat polymorphism upstream of rocA alters toxin production and enhances virulence in Streptococcus pyogenes. Infect Immun 2016, 84:2086-2093

14. Feng W, Minor D, Liu M, Li J, Ishaq SL, Yeoman C, Lei B: Null mutations of group A Streptococcus orphan kinase RocA: selection in mouse infection and comparison with CovS mutations in alteration of in vitro and in vivo protease $\mathrm{SpeB}$ expression and virulence. Infect Immun 2017, 85:e00790-16

15. Jain I, Miller EW, Danger JL, Pflughoeft KJ, Sumby P: RocA is an accessory protein to the virulence-regulating CovRS two- component system in group A Streptococcus. Infect Immun 2017, 85:e0274-17

16. Lynskey NN, Goulding D, Gierula M, Turner CE, Dougan G, Edwards RJ, Sriskandan S: RocA truncation underpins hyperencapsulation, carriage longevity and transmissibility of serotype M18 group A streptococci. PLoS Pathog 2013, 9:e1003842

17. Biswas I, Scott JR: Identification of rocA, a positive regulator of covR expression in the group A Streptococcus. J Bacteriol 2003, 185: 3081-3090

18. Lynskey NN, Velarde JJ, Finn MB, Dove SL, Wessels MR: RocA binds CsrS to modulate CsrRS-mediated gene regulation in group A Streptococcus. mBio 2019, 10:e01495-19

19. Sarkar P, Sumby P: Regulatory gene mutation: a driving force behind group a Streptococcus strain- and serotype-specific variation. Mol Microbiol 2017, 103:576-589 